

\title{
Swap-vertex based neighborhood for Steiner tree problems
}

\author{
Zhang-Hua Fu, Jin-Kao Hao
}

\section{To cite this version:}

Zhang-Hua Fu, Jin-Kao Hao. Swap-vertex based neighborhood for Steiner tree problems. Mathematical Programming Computation, 2017, 9(2) (212/311-2413123213310), pp.297-320. 10.1007/s12532016-0116-8 . hal-01532908v2

\section{HAL Id: hal-01532908 \\ https://hal.science/hal-01532908v2}

Submitted on 4 Jun 2017

HAL is a multi-disciplinary open access archive for the deposit and dissemination of scientific research documents, whether they are published or not. The documents may come from teaching and research institutions in France or abroad, or from public or private research centers.
L'archive ouverte pluridisciplinaire HAL, est destinée au dépôt et à la diffusion de documents scientifiques de niveau recherche, publiés ou non, émanant des établissements d'enseignement et de recherche français ou étrangers, des laboratoires publics ou privés. 


\title{
Swap-Vertex Based Neighborhood for Steiner Tree Problems
}

\author{
Zhang-Hua Fu • Jin-Kao Hao
}

Received: 09 April 2015 / Revised: 21 March, 03 Oct. 2016 / Accepted: 09 Dec. 2016 Mathematical Programming Computation DOI: 10.1007/s12532-016-0116-8

\begin{abstract}
Steiner tree problems (STPs) are very important in both theory and practice. In this paper, we introduce a powerful swap-vertex move operator which can be used as a basic element of any neighborhood search heuristic to solve many STP variants. Given the incumbent solution tree $T$, the swapvertex move operator exchanges a vertex in $T$ with another vertex out of $T$, and then attempts to construct a minimum spanning tree, leading to a neighboring solution (if feasible). We develop a series of dynamic data structures, which allow us to efficiently evaluate the feasibility of swap-vertex moves. Additionally, in order to discriminate different swap-vertex moves corresponding to the same objective value, we also develop an auxiliary evaluation function. We present a computational assessment based on a number of challenging problem instances (corresponding to three representative STP variants) which clearly shows the effectiveness of the techniques introduced in this paper. Particularly, as a key element of our KTS algorithm which participated in the 11th DIMACS implementation challenge, the swap-vertex operator as well as the auxiliary evaluation function contributed significantly to the excellent performance of our algorithm ${ }^{1}$.
\end{abstract}

Keywords Network design · Steiner tree problems · Swap-vertex move · Auxiliary evaluation function $\cdot 11$ th DIMACS implementation challenge

Zhang-Hua Fu

a) Institute of Robotics and Intelligent Manufacturing, The Chinese University of Hong Kong (Shenzhen), 518000, China

b) LERIA, Université d'Angers, 2 Boulevard Lavoisier, 49045 Angers Cedex 01, France

E-mail: fu@info.univ-angers.fr

Jin-Kao Hao (corresponding author)

a) LERIA, Université d'Angers, 2 Boulevard Lavoisier, 49045 Angers Cedex 01, France

b) Institut Universitaire de France, Paris, France

E-mail: jin-kao.hao@univ-angers.fr

1 The software that was reviewed as part of this submission has been issued with the Digital Object Identifier Doi:10.5281/zenodo.206998. 


\section{Introduction}

Many network design problems encountered in real-life applications (e.g., electricity, telecommunication, transportation, distribution supply, VLSI design, biology, signal processing, etc.) require to connect a number of basic equipments, with respect to various objectives and constraints [1]. These problems could generally be formulated as a class of broadly defined Steiner tree problems (STPs), where the optimal solutions have the structure of a tree. According to the pursued objectives and/or constraints to be considered, a number of STP variants can be defined, e.g., the classical STP [2], the rectilinear STP [3], the Euclidean STP [4], the prize-collecting STP [5], the node weighted STP [6] (including the maximum weight connected subgraph problem [7]), the group STP [8], the generalized STP [9], the obstacle avoiding STP [10], the directed STP [11], the dynamic STP [12], the stochastic STP [13], the STP with hop constraints [14], and mix of them $[15,16]$, etc. Notice that almost all these STP variants are known to be NP-hard [17-19] and thus computationally challenging.

Given the theoretical importance and practical relevance of the STPs, considerable effort has been put forth to develop both exact and heuristic methods during the last decades. On the one hand, numerous powerful exact algorithms (based on branch \& bound, branch \& cut, branch \& price, etc.) have been devised. These methods have the desirable property of guaranteeing to find the optimal solution if no time and space limit is imposed. In practice, the current best exact algorithms are able to achieve remarkable results on several well-studied STP variants [20-23]. On the other hand, due to the inherent computational complexity of STPs, in some cases, it is impossible for a method to enumerate effectively the solutions of a given instance. To handle problems whose optimal solutions cannot be reached within a reasonable time, heuristic and meta-heuristic algorithms, which aim to provide sub-optimal solutions within an acceptable time, have become the mainly applied methods. Actually, exact and heuristic methods complement each other and could be used to tackle different types of problems. These two approaches can also be combined to create even more powerful hybrid methods.

Among various heuristics for solving the STPs, local search or neighborhood search is certainly the most popular and effective approach [24-29]. Generally, local search relies on some key ingredients including most importantly one or several move (or transformation) operators responsible for generating neighboring solutions [30]. In the field of STPs, a couple of conventional move operators have already been developed. For instance, for the classical STP in graphs (SPG), four basic move operators, i.e., vertex insertion, vertex elimination, key-path exchange, key-vertex elimination are commonly used in the literature $[24,25]$, while for the prize-collecting STP (PCSPG), two move operators called vertex insertion and vertex elimination were developed in [27]. These move operators add or remove vertices and then try to reconstruct a new minimum spanning tree, since for both the SPG and the PCSPG, each feasible solution could be uniquely characterized by its spanned vertices set. 
Though the existing move operators are generally quite effective, additional improvements are still possible by introducing new move operators.

This paper is interested in designing a new and effective move operator, i.e., the swap-vertex move operator, which complements the existing move operators, and can be adopted by local search heuristics to solve a large class of STP variants. Given the incumbent solution tree $T$, the swap-vertex move operator exchanges a vertex in $T$ with another vertex out of $T$, and then tries to construct a minimum spanning tree (MST), resulting in a neighboring solution of $T$. This basic move operator realizes a natural operation and could be advantageously employed by heuristic methods. However, due to its unaffordable complexity in the general case, the power of this operator was not really explored by the existing heuristics designed for solving STPs.

In this work, we demonstrate that the swap-vertex move operator could be effectively applied to instances of various STP problems when 1) the optimal solution of the problem is necessarily a minimum spanning tree once the vertices to span are known, 2) the input graph has uniform edge costs (i.e., all the edges have the same cost) and 3) the input graph is of reasonable size ${ }^{2}$. This covers all the MWCS (the maximum weight connected subgraph problem) instances, as well as a number of particularly difficult SPG and PCSPG instances collected by the 11th DIMACS challenge (detailed in Section 4). More generally, as explained in [28], the proposed swap-vertex move operator can also be slightly adapted to solve problem instances with nearly uniform edge costs, even if its effectiveness might be somewhat impacted.

Another contribution of this work is a newly designed auxiliary evaluation function (defined in Section 3.3), based on the concept of special degree of a given solution. The auxiliary evaluation function aims to identify the most promising swap-vertex move among a number of candidate moves with the same move gain in terms of the objective function. The new function is used to guide the search algorithm towards a promising direction on search plateaus when the objective function alone cannot distinguish the candidate moves.

The remainder of this paper is organized as follows: After introducing three representative STP variants in Section 2, Section 3 presents the main idea as well as the technical details relative to the swap-vertex move operator. Section 4 reports experimental results on these three STP variants, in order to demonstrate the generality and effectiveness of the swap-vertex move operator. Finally, Section 5 is dedicated to concluding remarks.

\section{Problem Definitions}

To illustrate the main idea of the swap-vertex based neighborhood and the associated evaluation technique for Steiner tree problems, we choose, as illustrative examples, three representative STP variants: the classical Steiner tree

\footnotetext{
2 Depending on the available memory of the used computer, currently the swap-vertex move operator has been successfully applied to solve instances with up to 5226 vertices and 93394 edges, executed on a personal computer with 4GB RAM.
} 
problem in graphs $(\mathrm{SPG})$, the prize-collecting Steiner tree problem in graphs (PCSPG), and the maximum weight connected subgraph problem (MWCS). These STP variants are part of the 11th DIMACS implementation challenge [31] and attracted a large number of participants during the challenge.

2.1 Classical Steiner tree problem in graphs (SPG) [2]

Given an undirected graph $G=(V, E)$ with a set $V$ of vertices and a set $E$ of edges. The set $V$ is partitioned into two sets: a set of terminal vertices and a set of Steiner vertices. Each edge $e \in E$ has an associated cost $c_{e} \geq 0$. The SPG is to determine a subtree $T=\left(V_{T}, E_{T}\right)$ (with vertex set $V_{T}$ and edge set $E_{T}$ respectively) of $G$ spanning all terminal vertices and possibly some Steiner vertices, so as to minimize the total edge cost of the obtained tree, i.e.:

$$
\text { Minimize } f_{1}(T)=\sum_{e \in E_{T}} c_{e} .
$$

Specifically, the rectilinear Steiner tree problem [3] can be reduced to a special case of the SPG.

\subsection{Prize-collecting Steiner tree problem in graphs (PCSPG) [5]}

Given an undirected graph $G=(V, E)$ with vertex set $V$ and edge set $E$. Each vertex $v \in V$ is associated with a real-valued prize $p_{v} \geq 0$ (vertex $v$ is called a customer vertex if $p_{v}>0$, and a non-customer vertex otherwise), and each edge $e \in E$ is associated with a real-valued $\operatorname{cost} c_{e} \geq 0$. Then the PCSPG aims to find a subtree $T=\left(V_{T}, E_{T}\right)$ of $G$, so as to minimize the sum of the consumed edge costs plus the prizes of the vertices not spanned by $T$, i.e.:

$$
\text { Minimize } f_{2}(T)=\sum_{e \in E_{T}} c_{e}+\sum_{v \notin V_{T}} p_{v} .
$$

Note that the SPG is a special case of the PCSPG, if each terminal of the SPG is associated with a high enough prize (corresponding to a customer of the PCSPG), and each Steiner vertex is associated with a zero prize (corresponding to a non-customer vertex of the PCSPG).

Particularly, if an additional source vertex is chosen as the root which must be part of any feasible solution, we obtain a rooted version of the PCSPG (denoted by RPCST, another of the seven competition problems included in the 11th DIMACS challenge).

\subsection{Maximum-weight connected subgraph problem (MWCS) [7]}

Given an undirected graph $G=(V, E)$ with vertex set $V$ and edge set $E$. Each vertex is associated with a real-valued weight $w_{v}\left(w_{v}\right.$ might be negative), then 
the MWCS is to find a subset $V^{*} \subseteq V$ such that the induced graph over $V^{*}$ is connected and the total weight is maximized, i.e.:

$$
\text { Maximize } f_{3}(T)=\sum_{v \in V^{*}} w_{v} .
$$

In the MWCS, the edge costs are no longer under consideration, thus we should just guarantee connectivity of the induced graph. From the point of view of STPs, without changing the optimal solution, we can assume each edge has a zero cost, and the task is to find a subtree of graph $G$ such that the spanned vertices lead to the largest weight.

Using the rules described in [32], every PCSPG instance can be transformed to an equivalent MWCS instance, indicating that the MWCS is a basic model which potentially embraces all the four STP variants mentioned above.

\section{Swap-Vertex Based Neighborhood}

In this section, we present the general idea of the swap-vertex move operator and devise dedicated techniques for fast neighborhood evaluation. We also define an auxiliary evaluation function which aims to distinguish different swap-vertex moves corresponding to the same objective value.

\subsection{Main Idea}

According to the problem definitions, the above three STP variants (SPG, PCSPG and MWCS) share a common feature: the optimal solution must be a minimum spanning tree (MST) once the vertices to span are known. It indicates that any solution $T$ could be uniquely characterized by its spanned vertex set $V_{T}$. Accordingly, two basic move operators, which simply add (or remove) a vertex to (from) the incumbent solution (a MST) and then attempt to reconstruct a new MST, have been applied respectively to the SPG [25] and the PCSPG [27] (to our knowledge, no such basic move operator has been applied to the MWCS). Based on these two basic move operators, one can define two neighborhoods (denoted by $N_{1}(T), N_{2}(T)$ respectively) for a given incumbent solution $T$ with vertex set $V_{T}$ :

$$
\begin{aligned}
& N_{1}(T)=\left\{\operatorname{MST}\left(V_{T} \cup\{i\}\right), \forall i \notin V_{T}\right\}, \\
& N_{2}(T)=\left\{\operatorname{MST}\left(V_{T} \backslash\{i\}\right), \forall i \in V_{T}\right\} .
\end{aligned}
$$

where $\operatorname{MST}\left(V_{T} \cup\{i\}\right)$ and $\operatorname{MST}\left(V_{T} \backslash\{i\}\right)$ respectively denote the neighboring solution of $T$ achieved by adding a vertex $i$ to the vertex set $V_{T}$ of $T$ and removing $i$ from $V_{T}$.

The add and remove move operators are simple to implement and are widely adopted by local search heuristics. However, as we show in this work, the search performance of these two basic move operators could be considerably improved by introducing the swap-vertex move operator. 
Basically, given the incumbent solution $T$ (a MST) with its vertex set $V_{T}$, the swap-vertex move operator exchanges a vertex $i \in V_{T}$ with another vertex $j \notin V_{T}$, and then tries to reconstruct (dynamically) a new MST, leading to a neighboring solution of $T$ (possibly discarded if the new solution is infeasible). One notices that applying this move operator is equivalent to adding vertex $j$ and removing vertex $i$ simultaneously.

Accordingly, we can define the neighborhood $N_{3}(T)$ as follows:

$$
N_{3}(T)=\left\{\operatorname{MST}\left(V_{T} \cup\{j\} \backslash\{i\}\right), \forall i \in V_{T}, j \notin V_{T}\right\} .
$$

By combining this new neighborhood $N_{3}(T)$ with the two basic neighborhoods $N_{1}(T)$ and $N_{2}(T)$, a local search procedure could search within an enlarged neighborhood, thus increasing the opportunity of finding solutions of improved quality.

Although this idea is natural, the swap neighborhood has not been widely applied in existing STP local search methods, possibly due to the unreasonably high complexity needed for neighborhood exploration. Indeed, given the incumbent solution $T$ with its vertex set $V_{T}$, and let $n=|V|$ and $m=|E|$, then there are in total $O\left(\left|V_{T}\right|\right) \cdot O\left(|V|-\left|V_{T}\right|\right) \leq O\left(n^{2}\right)$ possible swap-vertex moves. If we choose to reconstruct a MST from scratch (e.g., using Kruskal's algorithm with the aid of a Fibonacci heap) after applying a swap-vertex move, the overall complexity would reach $O\left(n^{2}\right) \cdot O(m+n \cdot \log n)$, being unaffordable for mid- and large- sized instances.

Fortunately, for graphs with uniform edge costs, the above complexity could be reduced much. For example, in the MWCS problem which potentially covers many other STP variants, swapping a vertex $i \in V_{T}$ with a vertex $j \notin V_{T}$ (if feasible) would definitively increase the objective value by $\Delta=w_{j}-w_{i}(\Delta$ is called the move gain). Furthermore, for any SPG or PCSPG instance with uniform edge costs, feasibly swapping $i \in V_{T}$ with $j \notin V_{T}$ would never change the total consumed cost, indicating that the objective value would definitively increase by $\Delta=0$ (for the SPG) or $\Delta=p_{i}-p_{j}$ (for the PCSPG). In these cases, it is quite easy to calculate all the $O\left(n^{2}\right)$ possible $\Delta$ values, within an overall complexity of $O\left(n^{2}\right)$. However, to implement the swap operator effectively, we need to consider two additional questions: (1) How to verify the solution feasibility after swapping any pair of vertices? (2) How to distinguish the feasible moves leading to the same objective value? In the following subsections, we discuss in detail how to address these two questions.

\subsection{Feasibility Verification}

Given a solution $T$ with vertex set $V_{T}$ and edge set $E_{T}$, before swapping any pair of vertices (for the SPG, we only consider swapping Steiner vertices), we should verify at first its feasibility. Without loss of generality, we assume the incumbent solution $T$ has at least two vertices, then the feasibility after swapping each pair of vertices could be efficiently verified, with the aid of the 
following dynamic data structures (an illustrative example is provided at the end of this section).

- Step 1: For each vertex $i \in V_{T}$, remove $i$ from $V_{T}$ and remove the associated incidence edges from $E_{T}$, generally resulting in a number of disconnected components (step 1.1). Then, try to reconnect the components using edges between them (without leading to any cycle). The final solution after reconnection is typically a forest which is composed of a number of disconnected subtrees (step 1.2). After that, temporarily store the roots (dynamically determined during the calculation, relying on the dynamic data structures slightly adapted from [25]) of all the subtrees into a onedimensional array $A$ (step 1.3). Note that the length of $A$ (i.e., $|A|$ ) belongs to $\left[1, d_{i}\right]$, where $d_{i}$ is the degree of vertex $i$ in graph $G$.

Complexity: We briefly discuss the computational complexity of step 1 . At each iteration of local search, we just need to execute this step only once for each vertex $i \in V_{T}$, instead of running it repeatedly for each pair of vertices. Using dynamic updating techniques slightly adapted from the Steiner-vertex elimination move operator [25], at each iteration, the overall complexity needed to process all the vertices of $V_{T}$ (excluding step 1.3) could be bounded by $O(m \cdot \log n)$.

Given a forest corresponding to removing a vertex $i \in V_{T}$, one can obtain, with the aid of a union-find set data structure (dynamically updated), the root of each subtree within $O\left(d_{\max }\right)$ time, where $d_{\max }$ is the maximum possible degree of each vertex in graph $G$. Then, after removing each vertex $i \in V_{T}$, we need to obtain the roots of at most $r_{i}+1$ subtrees, where $r_{i}$ is the number of the children of vertex $i$ in solution $T$. This implies that at each iteration of local search, we should get the roots of at most $\sum_{i \in V_{T}}\left(r_{i}+1\right)=\sum_{i \in V_{T}} r_{i}+\left|V_{T}\right|=\left|V_{T}\right|-1+\left|V_{T}\right|<2 n$ subtrees. Consequently, step 1.3 can be finished within an overall complexity of $O\left(n \cdot d_{\max }\right)$.

- Step 2: For each vertex $j \notin V_{T}$ (for the sake of efficiency, here we only consider the vertices with at least one edge incident to solution $T$ ), examine all its adjacent vertices in $G$ and repeatedly check if a given adjacent vertex belongs to a subtree of the forest generated in step 1 . If this is the case, store (temporarily) the root of the corresponding subtree into another one-dimensional array $B$. Notice that array $B$ may have duplicates, and the length of $B$ (i.e., $|B|$ ) belongs to $\left[0, d_{j}\right]$, where $d_{j}$ is the degree of vertex $j$ in graph $G$.

Complexity: Unlike step 1, this step needs to be executed repeatedly for each pair of vertices, since the forests obtained in step 1 might be different corresponding to removing different vertices from $V_{T}$. As it is analyzed above, using union-find set (dynamically updated), getting the root of each subtree can be achieved within $O\left(d_{\max }\right)$, thus the complexity needed by 
step 2 for processing each pair of vertices (getting and storing the roots of at most $O\left(d_{\max }\right)$ subtrees) is bounded by $O\left(d_{\max }^{2}\right)$.

- Step 3: With the above two one-dimensional arrays $A$ (step 1 ) and $B$ (step 2), it is straightforward to verify the feasibility after swapping any pair of vertices. For this, it suffices to check if the root vertices stored in $B$ exactly cover the ones stored in $A$. If this is the case, the corresponding swap-vertex move is feasible, otherwise it is infeasible.

Complexity: This step can be finished within a complexity of $O(|A|+$ $|B|) \leq O\left(d_{\max }\right)$ for each pair of vertices.

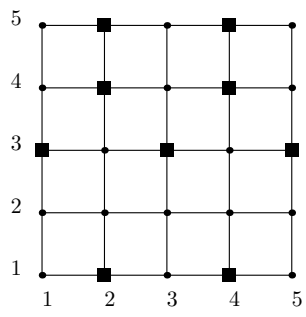

(a)

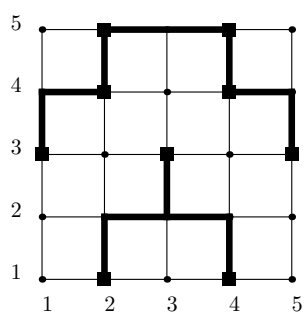

(d)

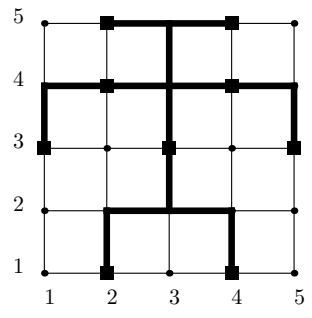

(b)

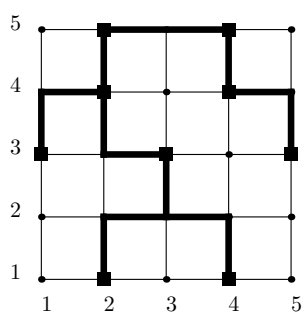

(e)

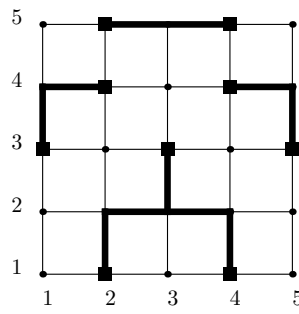

(c)

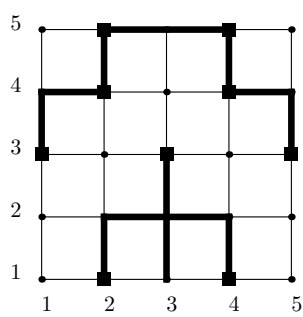

Fig. 1 Example showing how to verify feasibility after swapping any pair of vertices.

To summarize, the overall complexity needed for verifying the feasibility of all the $O\left(n^{2}\right)$ possible swap-vertex moves is bounded by $O\left(m \cdot \log n+n^{2} \cdot d_{\max }^{2}\right)$, being affordable for mid-sized graphs (even for large-sized sparse graphs with $O(m) \approx O(n)$, generally corresponding to a small value of $\left.d_{\max }\right)$.

Fig. 1 is a SPG example showing how to verify feasibility after swapping any pair of vertices. Sub-figure $(a)$ is the input graph with 25 vertices and 40 edges, where the 9 terminals are drawn in blocks and the 16 Steiner vertices are drawn in dots. For convenience, let $v_{x y}$ denote the vertex located at the cross-point of the $x$ th horizontal line and the $y$ th vertical line. Sub-figure $(b)$ is a feasible solution spanning all the 9 terminals and 7 Steiner vertices (assume it is rooted at vertex $v_{53}$ ). Now, we consider for example the feasibility after swapping vertex $v_{43}$ with any other un-spanned vertex. For this, we at first 
delete vertex $v_{43}$ and the associated edges, leading to a forest (sub-figure $(c)$ ), which includes 4 subtrees rooted at $v_{53}, v_{42}, v_{44}, v_{33}$ respectively. After that, we try to reconnect these subtrees using edges between them. As shown in sub-figure $(d)$, the subtrees rooted at $v_{53}, v_{42}, v_{44}$ could be merged into one subtree (rooted at $v_{53}$ ). Then the roots of the remaining two subtrees, i.e., $v_{53}$ and $v_{33}$, are stored into the one-dimensional array $A$.

With the above information, it is easy to verify the feasibility after swapping vertex $v_{43}$ with any other vertex. Consider vertex $v_{32}$ at first (similar for vertices $v_{34}, v_{21}$ and $v_{25}$ ), its adjacent vertices cover both subtrees rooted at $v_{53}$ and $v_{33}$, thus leading to a feasible solution (sub-figure $(e)$ ). By contrast, for vertex $v_{13}$, its adjacent vertices only cover one subtree (the subtree rooted at $v_{33}$ ), meaning that swapping $v_{43}$ and $v_{13}$ would lead to an infeasible solution (sub-figure $(f))$. This case is similar for vertices $v_{11}, v_{51}, v_{15}$ and $v_{55}$.

\subsection{Evaluation Function}

Among all the feasible swap-vertex moves, there are generally a number of moves with the same move gain, which need to be further distinguished in order for the search to identify the most promising moves. For example, in a SPG instance with uniform edge costs, although swapping any two Steiner vertices would never change the objective value (thus leads to the same zero move gain), it is still possible that some swap-vertex moves might be more promising than others in the sense that they lead to a solution which could be further improved by the search algorithm. However, the initial objective function alone cannot allow us to identify the most promising swap-vertex moves. In order to overcome this difficulty, we design a more discriminating evaluation function which aims to guide the search towards promising solutions, inspired by our previous experiences on graph labeling problems [33,34].

Let us consider a SPG instance with uniform edge costs at first. In this case, feasibly removing a Steiner vertex would definitively improve the objective value. Now, let $T$ be a solution where no Steiner vertex could be feasibly removed. Although swapping any Steiner vertex in $T$ and another vertex out of $T$ would never change the objective value, it may become possible again to feasibly remove some Steiner vertex, thus further improving the objective value (see the example of Fig. 2 and the explanations given at the end of this section). This observation could be similarly extended to the PCSPG and the MWCS. Respectively, in any PCSPG instance with uniform edge costs, feasibly removing a vertex with prize lower than the cost of each edge would certainly improve the objective value. In any MWCS instance, feasibly removing a vertex with negative weight would definitively lead to an improving solution (regardless of the edge costs).

Inspired by these observations, for the three STP variants studied in this paper, we define an auxiliary evaluation function as follows, which aims to estimate the opportunity of feasibly removing vertices. 
Definition 1. Given the incumbent solution $T$ with vertex set $V_{T}$, for each vertex $i \in V_{T}$, its special degree $s d_{i}$ is defined as the number of vertices belonging to $V_{T}$ which are directly reachable from $i$ (we say a vertex $j \neq i$ is directly reachable from vertex $i$ if edge $(i, j) \in E$ ), i.e.,:

$$
s d_{i}=\sum_{j \in V_{T}, j \neq i,(i, j) \in E} 1 .
$$

According to this definition, we observe that if $s d_{i}=1$, it means vertex $i$ is directly reachable from only one other vertex $j \in V_{T}$, implying that once the related vertex $j$ is removed from $V_{T}$, the resulting solution would become disconnected (unless $\left|V_{T}\right|=2$ ).

Definition 2. The special degree $\operatorname{sd}(T)$ of a feasible solution $T$ is defined as the number of vertices with special degree $s d_{i}=1$, i.e., :

$$
s d(T)=\sum_{i \in V_{T}, s d_{i}=1} 1 .
$$

The values of $s d(T)$ could be dynamically evaluated, within a complexity of $O\left(d_{\max }\right)$ after swapping each pair of vertices.

Intuitively, the lower the value of $s d(T)$, the larger the opportunity to feasibly remove a vertex (thus the larger the opportunity to further improve the objective value). For each of the three STP variants studied in this paper, to identify improving solutions, we use the objective value defined in Section 2 as the main evaluation criterion, and adopt the special degree $s d(T)$ as an auxiliary evaluation criterion. Precisely, we say solution $T 1$ dominates solution $T 2$ if 1) the objective value of $T 1$ is better than that of $T 2$, or 2) $T 1$ and $T 2$ have the same objective value, but $T 1$ has a special degree lower than $T 2$.

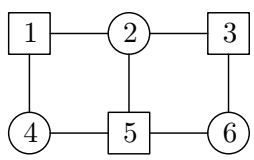

(a)

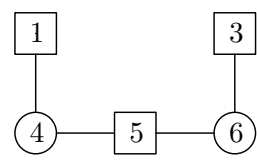

(b)



(c)

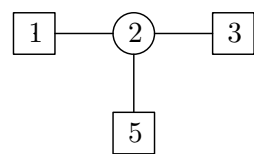

(d)

Fig. 2 Example of applying the swap-vertex move guided by the auxiliary evaluation function.

Fig. 2 is a SPG example (with $c_{e}=1, \forall e \in E$ ) which shows the search process of applying the swap-vertex move operator, guided by the auxiliary evaluation function. Respectively, sub-figure $(a)$ is the input graph with three terminals (vertex 1, 3, 5, drawn in boxes) and three Steiner vertices (vertex $2,4,6$, drawn in circles). Sub-figure $(b)$ is an initial solution with objective value $f(b)=4$. Notice that solution $(b)$ could not be further improved by simply adding or removing any Steiner vertex. However, if we swap vertex 6 with vertex 2 to get a new solution $(c)$, although the objective value does not vary, the special degree decreases. More precisely, $s d(b)=2$ (both vertex 1 
and 3 have a special degree $s d_{1}=s d_{3}=1$ ), and $s d(c)=1$ (only vertex 3 has a special degree $s d_{3}=1$ ). Now, from solution $(c)$, we could feasibly remove Steiner vertex 4 , to obtain an improving solution $(d)$ with objective value $f(d)=3$. Obviously, the swap-vertex move operator as well as the auxiliary evaluation function play a crucial role during this search process.

\section{Experimental Results}

This section is dedicated to a computational assessment of the proposed swapvertex move operator as well as the associated auxiliary evaluation function. This study is based on a set of representative benchmark instances for each of the three studied STP variants (SPG, PCSPG and MWCS).

\subsection{Experimental Protocol}

In order to particularly emphasize the importance of the swap-vertex move operator as well as the auxiliary evaluation function, we avoid to test them within a complex algorithm. Instead, we implement and compare three simple local search procedures where the search is fully driven by the employed neighborhoods and evaluation functions. We respectively call these three procedures 'Basic-LS' (with add and remove moves of Section 3.1, but without the swap-vertex move described in Section 3.1), 'Swap-LS' (Basic-LS reinforced by the swap-vertex move, but without the auxiliary evaluation function defined in Section 3.3), and 'Enhanced-LS' (Basic-LS reinforced by the swap-vertex move and the auxiliary evaluation function). All these three procedures were coded in the $\mathrm{C}$ language ${ }^{3}$, shared the same data structures whenever it is possible and were executed on the same computing platform, i.e., a machine with an Intel(R) Core(TM) i5-4460 3.20GHz processor with 4 cores and 4GB RAM. Note that each job occupied only one core, being executed in sequential order.



3 Our source codes are available at: http://www.info.univ-angers.fr/pub/hao/SPG_ SWAP/CODE.zip 
- Basic-LS (Algorithm 1): Starting from a given initial solution T, Basic-LS examines in random order the solutions of $N_{1}(T) \cup N_{2}(T)$, i.e., the union of the two neighborhoods induced by the add and remove operators (defined in Section 3.1, using the dynamic updating techniques described in [25] for efficient evaluations), and iteratively replaces the incumbent solution with the first met improving solution (only regarding the objective value). The process stops when no improving solution exists in the whole neighborhood, meaning that a local optimum is reached.

- Swap-LS (Algorithm 2): Swap-LS is an improved version of Basic-LS. It realizes the search with an enlarged neighborhood $N_{1}(T) \cup N_{2}(T) \cup N_{3}(T)$, i.e., the union of the three neighborhoods induced by the add, remove and swap-vertex operators (defined in Section 3.1).

- Enhanced-LS (Algorithm 3): Enhanced-LS is the same as Swap-LS with the following difference. Enhanced-LS uses, instead of the original objective function, the auxiliary evaluation function described in Section 3.3 to evaluate the perspective of each neighboring solution.

We did not implement and compare a variant with the conventional move operators (add and remove) combined with the auxiliary evaluation function. In fact, without the swap-vertex move operator, the auxiliary evaluation function is of little interest since in general adding or removing a vertex will change the objective value.

Additionally, the above three algorithms use the following heuristic for generating initial solutions (uniform for all the three studied STP variants). We call a terminal vertex (for the SPG), or a vertex with positive prize (for the PC-

SPG) or positive weight (for the MWCS) as a candidate vertex. Then, starting from a randomly selected candidate vertex, we try to complete the incumbent solution by inserting a new path connecting a randomly chosen un-spanned candidate vertex (for the PCSPG and the MWCS, we should guarantee that the objective value after insertion is improved). This process is repeated until no further path can be inserted, meaning that an initial solution is generated.

In the following subsections, we compare the performances of the three algorithms on the SPG, the PCSPG and the MWCS, using a set of representative benchmarks for each problem. To ensure fair comparisons, for each test instance, we repeatedly ran each algorithm, each run independently restarting from a randomly generated initial solution. This process was repeated until one CPU hour was reached.

Finally, we also briefly summarize the competition results of the 11th DIMACS implementation challenge, in order to provide some supplementary information about the effectiveness of the techniques introduced in this paper.

\subsection{Results on the SPG}

We chose 25 SPG instances (the un-weighted instances of types hc, bip and cc) from the 11th DIMACS challenge as benchmarks to evaluate the performances of the three algorithms. These instances are all with uniform edge costs and of reasonable size $(|V|<6000)$, thus being suitable to apply the swap-vertex move operator. Moreover, they are known to be extremely difficult for the existing approaches such that most of these instances still remain open.

The detailed results are summarized in Table 1, where the first two columns indicate the instance name and the corresponding optimal result (extracted from [20], if applicable). The next two columns respectively indicate the overall best objective value found by Basic-LS (indicated with a symbol "*" if it reaches the known optimal result), and the average objective value of each independent run. For comparisons, columns 5 and 6 indicate the same information corresponding to Swap-LS, where the objective values better than those of Basic-LS are indicated in bold, and the same objective values are indicated in italic. Additionally, column 7 gives the mean improvement percentage obtained by Swap-LS compared to Basic-LS, in terms of best objective values. Finally, we give in the last three columns similar information corresponding to Enhanced-LS.

In terms of best objective values, compared to Basic-LS, Swap-LS obtains within the same allowed time (one CPU hour for each instance) 4 better, 6 worse and 15 equal results, corresponding to a mean deterioration of $0.11 \%$ (averaged on all these 25 instances) provided by Swap-LS. Under the same test condition, compared to Basic-LS, Enhanced-LS obtains 20 better results and yields the same results on the remaining 5 instances (all reaching optimality), leading to a mean improvement of $3.93 \%$. Furthermore, we used the Friedman test to check the statistical differences between the best objective values of the compared algorithms, which respectively reveals a $p$-value of 
Table 1 Comparative results between Basic-LS, Swap-LS and Enhanced-LS on 25 representative SPG instances (within one CPU hour)

\begin{tabular}{|c|c|c|c|c|c|c|c|c|c|}
\hline \multirow{2}{*}{ Instance } & \multirow{2}{*}{ Optimal } & \multicolumn{2}{|c|}{ Basic-LS } & \multicolumn{3}{|c|}{ Swap-LS } & \multicolumn{3}{|c|}{ Enhanced-LS } \\
\hline & & $f^{\text {best }}$ & $f^{a v g}$ & $f^{\text {best }}$ & $f^{a v g}$ & Improve & $f^{\text {best }}$ & $f^{\text {avg }}$ & Improve \\
\hline hc6u.stp & 39.00 & $39.00^{*}$ & 43.80 & $39.00 *$ & 43.79 & $0.00 \%$ & $39.00^{*}$ & 39.28 & $0.00 \%$ \\
\hline hc7u.stp & 77.00 & $77.00 *$ & 87.63 & $77.00 *$ & 87.63 & $0.00 \%$ & $77.00 *$ & 77.30 & $0.00 \%$ \\
\hline hc8u.stp & 148.00 & 153.00 & 173.86 & 153.00 & 173.89 & $0.00 \%$ & $148.00 *$ & 150.84 & $3.27 \%$ \\
\hline hc9u.stp & - & 304.00 & 345.92 & 307.00 & 345.84 & $-0.99 \%$ & 293.00 & 298.37 & $3.62 \%$ \\
\hline hc10u.stp & - & 624.00 & 687.27 & 623.00 & 687.21 & $0.16 \%$ & 586.00 & 591.30 & $6.09 \%$ \\
\hline hc11u.stp & - & 1268.00 & 1366.94 & 1270.00 & 1366.88 & $-0.16 \%$ & 1169.00 & 1173.97 & $7.81 \%$ \\
\hline hc12u.stp & - & 2593.00 & 2720.42 & 2569.00 & 2721.86 & $0.93 \%$ & 2321.00 & 2333.92 & $10.49 \%$ \\
\hline bip 42 u.stp & 236.00 & 258.00 & 264.02 & 258.00 & 264.02 & $0.00 \%$ & 239.00 & 243.48 & $7.36 \%$ \\
\hline bip52u.stp & - & 263.00 & 268.05 & 264.00 & 268.05 & $-0.38 \%$ & 238.00 & 241.22 & $9.51 \%$ \\
\hline bip62u.stp & - & 235.00 & 239.83 & 234.00 & 239.83 & $0.43 \%$ & 221.00 & 223.87 & $5.96 \%$ \\
\hline bipa2u.stp & - & 374.00 & 377.72 & 374.00 & 377.72 & $0.00 \%$ & 343.00 & 347.35 & $8.29 \%$ \\
\hline bipe2u.stp & 54.00 & 55.00 & 57.00 & 55.00 & 57.00 & $0.00 \%$ & $54.00^{*}$ & 54.88 & $1.82 \%$ \\
\hline cc3-4n.stp & 13.00 & $13.00 *$ & 13.40 & $13.00 *$ & 13.40 & $0.00 \%$ & $13.00^{*}$ & 13.00 & $0.00 \%$ \\
\hline cc3-5n.stp & 20.00 & $20.00 *$ & 21.77 & $20.00 *$ & 21.77 & $0.00 \%$ & $20.00 *$ & 20.07 & $0.00 \%$ \\
\hline cc3-10n.stp & - & 76.00 & 89.08 & 76.00 & 89.08 & $0.00 \%$ & 75.00 & 75.57 & $1.32 \%$ \\
\hline cc3-11n.stp & - & 93.00 & 109.25 & 94.00 & 109.25 & $-1.08 \%$ & 92.00 & 92.32 & $1.08 \%$ \\
\hline cc3-12n.stp & - & 113.00 & 133.47 & 115.00 & 133.47 & $-1.77 \%$ & 111.00 & 111.70 & $1.77 \%$ \\
\hline cc5-3n.stp & 42.00 & 44.00 & 49.20 & 44.00 & 49.20 & $0.00 \%$ & 43.00 & 46.17 & $2.27 \%$ \\
\hline cc6-2n.stp & 18.00 & $18.00^{*}$ & $\begin{array}{l}19.86 \\
19.86\end{array}$ & $\begin{array}{l}44.000 * \\
18.00 *\end{array}$ & 19.86 & $0.00 \%$ & $18.00^{*}$ & 18.00 & $0.00 \%$ \\
\hline cc6-3n.stp & 100.00 & 106.00 & 114.88 & 106.00 & 114.88 & $0.00 \%$ & 104.00 & 109.08 & $1.89 \%$ \\
\hline cc7-3n.stp & - & 318.00 & 332.67 & 318.00 & 332.67 & $0.00 \%$ & 298.00 & 305.05 & $6.29 \%$ \\
\hline cc9-2n.stp & - & 106.00 & 113.80 & 106.00 & 113.79 & $0.00 \%$ & 102.00 & 107.26 & $3.77 \%$ \\
\hline cc10-2n.stp & - & 194.00 & 205.36 & 195.00 & 205.36 & $-0.52 \%$ & 185.00 & 189.96 & $4.64 \%$ \\
\hline cc11-2n.stp & - & 358.00 & 372.90 & 358.00 & 372.90 & $0.00 \%$ & 340.00 & 348.71 & $5.03 \%$ \\
\hline cc12-2n.stp & - & 679.00 & 696.14 & 675.00 & 696.09 & $0.59 \%$ & 639.00 & 645.89 & $5.89 \%$ \\
\hline Average & - & - & - & - & - & $-0.11 \%$ & - & - & $3.93 \%$ \\
\hline
\end{tabular}

$9.39 \times 10^{-1}$ (between Swap-LS and Basic-LS), a $p$-value of $3.75 \times 10^{-6}$ (between Enhanced-LS and Basic-LS), and a $p$-value of $3.57 \times 10^{-7}$ (between EnhancedLS and Swap-LS). These results indicate that, in terms of best objective values, Enhanced-LS performs clearly better than Basic-LS and Swap-LS, while there is no significant difference between Swap-LS and Basic-LS.

In terms of average objective values, compared to Basic-LS, Swap-LS obtains 6 better, 2 worse and 17 equal results, while Enhanced-LS succeeds to obtain a better result on each of the 25 test instances. The Friedman test in terms of average objective values respectively reveals a $p$-value of $8.09 \times 10^{-1}$ (between Swap-LS and Basic-LS), a $p$-value of $3.69 \times 10^{-9}$ (between EnhancedLS and Basic-LS), and a $p$-value of $9.07 \times 10^{-8}$ (between Enhanced-LS and Swap-LS), indicating again that Enhanced-LS is a much improved version over Basic-LS and Swap-LS, while Swap-LS does not perform significantly differently from Basic-LS.

To conclude, the swap-vertex move operator alone does not perform well enough on the SPG instances with uniform edge costs (on these instances swapping any two Steiner vertices would never change the objective value). In contrast, when it is combined with the auxiliary evaluation function, we obtain a highly competitive algorithm to solve the SPG instances with uniform edge costs.

\subsection{Results on the PCSPG}

For the PCSPG, we chose 40 benchmark instances with uniform edge costs from the 11th DIMACS challenge, including the un-weighted instances of groups $\mathrm{H}, \mathrm{H} 2$, and all the instances of groups PUCNU, ACTMODPC (we do not use the HAND instances since they are too large to apply the swap- 
Table 2 Comparative results between Basic-LS, Swap-LS and Enhanced-LS on 40 representative PCSPG instances (within one CPU hour)

\begin{tabular}{|c|c|c|c|c|c|c|c|c|c|}
\hline \multirow[t]{2}{*}{ Instance } & \multirow[t]{2}{*}{ Optimal } & \multicolumn{2}{|c|}{ Basic-LS } & \multicolumn{3}{|c|}{ Swap-LS } & \multicolumn{3}{|c|}{ Enhanced-LS } \\
\hline & & $f^{\text {best }}$ & $f^{a v g}$ & $f^{\text {best }}$ & $f^{a v g}$ & Improve & $f^{\text {best }}$ & $f^{a v g}$ & Improve \\
\hline hc6u.stp & 36.00 & $36.00^{*}$ & 38.77 & $36.00^{*}$ & 38.77 & $0.00 \%$ & $36.00^{*}$ & 37.04 & $0.00 \%$ \\
\hline hc7u.stp & 72.00 & 73.00 & 77.62 & 73.00 & 77.61 & $0.00 \%$ & 72.00* & 73.60 & $1.37 \%$ \\
\hline hc8u.stp & 143.00 & 146.00 & 154.14 & 146.00 & 154.15 & $0.00 \%$ & $143.00 *$ & 145.83 & $2.05 \%$ \\
\hline hc9u.stp & 283.00 & 295.00 & 310.53 & 295.00 & 310.51 & $0.00 \%$ & 284.00 & 289.56 & $3.73 \%$ \\
\hline hc10u.stp & - & 588.00 & 609.66 & 588.00 & 609.64 & $0.00 \%$ & 565.00 & 571.15 & $3.91 \%$ \\
\hline hc11u.stp & - & 1184.00 & 1216.96 & 1178.00 & 1216.87 & $0.51 \%$ & 1131.00 & 1140.03 & $4.48 \%$ \\
\hline hc12u.stp & - & 2381.00 & 2426.55 & 2378.00 & 2427.14 & $0.13 \%$ & 2255.00 & 2266.11 & $5.29 \%$ \\
\hline hc6u2.stp & 20.00 & $20.00 *$ & 21.54 & $20.00 *$ & 21.55 & $0.00 \%$ & $20.00 *$ & 20.12 & $0.00 \%$ \\
\hline hc7u2.stp & 47.00 & $47.00^{*}$ & 51.16 & $47.00 *$ & 51.16 & $0.00 \%$ & $47.00 *$ & 47.66 & $0.00 \%$ \\
\hline hc8u2.stp & 97.00 & 99.00 & 104.36 & 98.00 & 104.35 & $1.01 \%$ & $97.00^{*}$ & 99.39 & $2.02 \%$ \\
\hline hc9u2.stp & - & 196.00 & 204.81 & 196.00 & 204.79 & $0.00 \%$ & 190.00 & 193.84 & $3.06 \%$ \\
\hline hc10u2.stp & - & 398.00 & 414.90 & 398.00 & 414.93 & $0.00 \%$ & 383.00 & 387.66 & $3.77 \%$ \\
\hline hc11u2.stp & - & 799.00 & 818.22 & 799.00 & 818.21 & $0.00 \%$ & 761.00 & 768.20 & $4.76 \%$ \\
\hline hc12u2.stp & - & 1602.00 & 1631.39 & 1602.00 & 1631.64 & $0.00 \%$ & 1516.00 & 1524.11 & $5.37 \%$ \\
\hline bip42nu.stp & 226.00 & 244.00 & 251.08 & 244.00 & 251.08 & $0.00 \%$ & 228.00 & 231.59 & $6.56 \%$ \\
\hline bip52nu.stp & 222.00 & 246.00 & 250.07 & 246.00 & 250.06 & $0.00 \%$ & 224.00 & 226.66 & $8.94 \%$ \\
\hline bip62nu.stp & 214.00 & 227.00 & 232.26 & 227.00 & 232.26 & $0.00 \%$ & 215.00 & 217.91 & $5.29 \%$ \\
\hline bipa2nu.stp & & 358.00 & 363.63 & 357.00 & 363.64 & $0.28 \%$ & 327.00 & 330.76 & $8.66 \%$ \\
\hline bipe2nu.stp & 53.00 & 54.00 & 55.04 & 54.00 & 55.05 & $0.00 \%$ & $53.00^{*}$ & 53.18 & $1.85 \%$ \\
\hline cc3-4nu.stp & 10.00 & $10.00 *$ & 10.90 & $10.00 *$ & 10.90 & $0.00 \%$ & $10.00 *$ & 10.59 & $0.00 \%$ \\
\hline cc3-5nu.stp & 17.00 & $17.00 *$ & 18.52 & $17.00^{*}$ & 18.52 & $0.00 \%$ & $17.00 *$ & 17.96 & $0.00 \%$ \\
\hline cc3-10nu.stp & - & 62.00 & 65.63 & 62.00 & 65.62 & $0.00 \%$ & 62.00 & 63.77 & $0.00 \%$ \\
\hline cc3-11nu.stp & - & 86.00 & 89.96 & 86.00 & 89.96 & $0.00 \%$ & 85.00 & 86.55 & $1.16 \%$ \\
\hline cc3-12nu.stp & - & 99.00 & 107.45 & 99.00 & 107.45 & $0.00 \%$ & 98.00 & 103.34 & $1.01 \%$ \\
\hline cc5-3nu.stp & 36.00 & 37.00 & 38.29 & 37.00 & 38.29 & $0.00 \%$ & 37.00 & 38.23 & $0.00 \%$ \\
\hline cc6-2nu.stp & 15.00 & $15.00 *$ & 15.23 & $15.00 *$ & 15.23 & $0.00 \%$ & $15.00 *$ & 15.23 & $0.00 \%$ \\
\hline cc6-3nu.stp & 95.00 & 98.00 & 104.48 & 98.00 & 104.47 & $0.00 \%$ & 97.00 & 103.46 & $1.02 \%$ \\
\hline cc7-3nu.stp & - & 288.00 & 298.27 & 288.00 & 298.30 & $0.00 \%$ & 279.00 & 287.80 & $3.13 \%$ \\
\hline cc9-2nu.stp & 83.00 & 85.00 & 89.20 & 85.00 & 89.20 & $0.00 \%$ & 84.00 & 88.91 & $1.18 \%$ \\
\hline cc10-2nu.stp & - & 173.00 & 182.17 & 174.00 & 182.15 & $-0.58 \%$ & 172.00 & 179.92 & $0.58 \%$ \\
\hline cc11-2nu.stp & - & 320.00 & 332.89 & 321.00 & 332.87 & $-0.31 \%$ & 312.00 & 325.05 & $2.50 \%$ \\
\hline cc12-2nu.stp & - & 597.00 & 613.06 & 598.00 & 612.68 & $-0.17 \%$ & 583.00 & 597.81 & $2.35 \%$ \\
\hline HCMV.stp & 7371.54 & 7373.39 & 7382.61 & 7373.39 & 7379.61 & $0.00 \%$ & 7373.39 & 7379.61 & $0.00 \%$ \\
\hline lymphoma.stp & 3341.89 & 3371.24 & 3412.78 & 3371.24 & 3406.95 & $0.00 \%$ & 3371.24 & 3406.96 & $0.00 \%$ \\
\hline metabol_expr_mice_1.stp & 11346.93 & 11485.55 & 11838.56 & 11485.06 & 611834.59 & $0.00 \%$ & 11485.06 & 311835.06 & $30.00 \%$ \\
\hline metabol_expr_mice_2.stp & 16250.24 & 16342.56 & 16495.46 & 16342.56 & 16493.85 & $50.00 \%$ & 16342.56 & 16493.82 & $20.00 \%$ \\
\hline metabol_expr_mice_3.stp & 16919.62 & 17173.26 & 17408.79 & 17159.42 & 217406.32 & $0.08 \%$ & 17159.42 & 217406.29 & $0.08 \%$ \\
\hline drosophila001.stp & 8273.98 & 8286.43 & 8300.51 & 8286.43 & 8297.74 & $0.00 \%$ & 8286.43 & 8297.74 & $0.00 \%$ \\
\hline drosophila005.stp & 8121.31 & 8211.42 & 8268.89 & 8197.07 & 8261.44 & $0.17 \%$ & 8195.64 & 8261.72 & $0.19 \%$ \\
\hline drosophila0075.stp & 8039.86 & 8133.80 & 8210.47 & 8120.13 & 8202.67 & $0.17 \%$ & 8122.07 & 8201.17 & $0.14 \%$ \\
\hline Average & & & & & & $0.03 \%$ & & & $2.11 \%$ \\
\hline
\end{tabular}

vertex move operator). These instances are also very challenging since many of them still remain unsolved. The corresponding optimal results are also extracted from [20] (if known). Like before, for each instance, we repeatedly ran Basic-LS (respectively, Swap-LS and Enhanced-LS) for one CPU hour, and show the obtained results in Table 2, with the same information as in Table 1.

Table 2 discloses that, in terms of best objective values, Swap-LS obtains within the same allowed time 8 better, 3 worse and 29 equal results compared to Basic-LS, corresponding to a mean improvement of $0.03 \%$. For comparison, Enhanced-LS obtains 28 better and 12 equal results compared to Basic-LS, leading to a mean improvement of $2.11 \%$. Furthermore, the Friedman test in terms of best objective values reveals a $p$-value of $5.60 \times 10^{-1}$ (between Swap-LS and Basic-LS), a $p$-value of $2.71 \times 10^{-9}$ (between Enhanced-LS and Basic-LS), and a $p$-value of $6.09 \times 10^{-7}$ (between Enhanced-LS and Swap-LS), indicating again that Enhanced-LS performs clearly better than Basic-LS and Swap-LS, while there is no significant difference between Swap-LS and Basic-LS.

In terms of average objective values, compared to Basic-LS, Swap-LS obtains 22 better, 8 worse and 10 equal results. In contrast, Enhanced-LS obtains a better average result on 39 out of the 40 test instances, only with one exception on which Enhanced-LS yields a same average result. The Fried- 
man test in terms of average objective values respectively reveals a $p$-value of $1.11 \times 10^{-1}$ (between Swap-LS and Basic-LS), a $p$-value of $1.73 \times 10^{-12}$ (between Enhanced-LS and Basic-LS), and a $p$-value of $7.02 \times 10^{-7}$ (between Enhanced-LS and Swap-LS), indicating that Swap-LS performs slightly better than Basic-LS (especially on the last eight instances of group ACTMODPC with quite different structures), while Enhanced-LS performs overall clearly better than both Basic-LS and Swap-LS.

This analysis showed that on most of the PCSPG instances with uniform edge costs, it is very useful to combine the swap-vertex move operator with the auxiliary evaluation function within a local-search based algorithm.

\subsection{Results on the MWCS}

Now we turn our attention to the more general MWCS, for which any instance of reasonable size is suitable to apply the swap-vertex move operator (there are no edge costs for the MWCS). More importantly, as explained in Section 2 , the MWCS is a basic model which potentially covers many related STP variants (including both the SPG and the PCSPG), indicating the generality of the swap-vertex move operator for tackling various STP variants.

We chose as benchmarks the 32 representative MWCS instances which were adopted as the final test set by the 11th DIMACS challenge. Similarly, for each of these 32 instances, we repeatedly and independently ran Basic-LS, Swap-LS and Enhanced-LS with a cutoff limit of one CPU hour. The obtained results are listed in Table 3, where we display the same statistics as in the previous tables. As indicated in column 2, all these instances have known optimal results $[20,23]$.

As shown in Table 3, in terms of best objective values, within the same allowed time, Swap-LS obtains 19 better, 1 worse and 12 equal results compared to Basic-LS, corresponding to a mean improvement of $2.09 \%$. Under the same test condition, Enhanced-LS also obtains 19 better, 1 worse and 12 equal results compared to Basic-LS, leading to a mean improvement of $2.20 \%$. Respectively, the Friedman test in terms of best objective values reveals a $p$ value of $1.75 \times 10^{-5}$ (between Swap-LS and Basic-LS), a $p$-value of $7.77 \times 10^{-6}$ (between Enhanced-LS and Basic-LS) and a $p$-value of $9.84 \times 10^{-1}$ (between Enhanced-LS and Swap-LS), indicating that there is no significant difference between Enhanced-LS and Swap-LS, while they both clearly dominate BasicLS.

In terms of average objective values, compared to Basic-LS, Swap-LS and Enhanced-LS both obtains 29 better and 3 equal results, while the Friedman test respectively reveals a $p$-value of $1.70 \times 10^{-6}$ (between Swap-LS and BasicLS), a $p$-value of $4.10 \times 10^{-10}$ (between Enhanced-LS and Basic-LS) and a $p$-value of $3.46 \times 10^{-1}$ (between Enhanced-LS and Swap-LS), indicating again that Enhanced-LS and Swap-LS performs similarly, while they both perform clearly better than Basic-LS. 
Table 3 Comparative results between Basic-LS, Swap-LS and Enhanced-LS on 32 representative MWCS instances (within one CPU hour)

\begin{tabular}{|c|c|c|c|c|c|c|c|c|c|}
\hline \multirow[t]{2}{*}{ Instance } & \multirow[t]{2}{*}{ Optimal } & \multicolumn{2}{|c|}{ Basic-LS } & \multicolumn{3}{|c|}{ Swap-LS } & \multicolumn{3}{|c|}{ Enhanced-LS } \\
\hline & & $f^{b e s t}$ & $f^{a v g}$ & $f^{\text {best }}$ & $f^{a v g}$ & Improve & $f^{\text {best }}$ & $f^{a v g}$ & Improve \\
\hline drosophila001.stp & 24.4 & 11.9 & 3.27 & 11.9 & 3.31 & $0.00 \%$ & 11.9 & 3.31 & $0.00 \%$ \\
\hline drosophila005.stp & 178.7 & 150.3 & 109.02 & 165.3 & 120.52 & $9.93 \%$ & 165.3 & 119.59 & $9.97 \%$ \\
\hline drosophila0075.stp & 260.5 & 231.8 & 211.88 & 246.4 & 222.25 & $6.29 \%$ & 243.1 & 227.04 & $4.87 \%$ \\
\hline HCMV.stp & 7.6 & 5.0 & 0.64 & 5.0 & 0.64 & $0.00 \%$ & 5.0 & 0.64 & $0.00 \%$ \\
\hline lymphoma.stp & 70.2 & 61.1 & 31.55 & 60.9 & 32.72 & $-0.39 \%$ & 60.9 & 32.69 & $-0.39 \%$ \\
\hline metabol_expr_mice_1.stp & 544.9 & 533.1 & 411.17 & 538.6 & 418.53 & $1.02 \%$ & 537.5 & 421.65 & $0.82 \%$ \\
\hline metabol_expr_mice_2.stp & 241.1 & $241.1 *$ & 114.44 & $241.1^{*}$ & 116.46 & $0.00 \%$ & $241.1^{*}$ & 116.49 & $0.00 \%$ \\
\hline metabol_expr_mice_3.stp & 508.3 & 477.3 & 308.11 & 495.8 & 320.12 & $3.88 \%$ & 495.8 & 319.77 & $3.88 \%$ \\
\hline 1500-a-0_6-d-0_25-e-0_25.stp & 1333.5 & 1193.6 & 1118.52 & 1255.5 & 1205.98 & $3.18 \%$ & 1261.3 & 1218.59 & $5.66 \%$ \\
\hline 1500-a-0_6-d-0_25-e-0_5.stp & 2799.7 & 2787.2 & 2748.63 & $2799.7^{*}$ & 2788.98 & $0.45 \%$ & $2799.7 *$ & 2792.24 & $0.45 \%$ \\
\hline 750-a-0_647-d-0_25-e-0_25.stp & 702.6 & 672.9 & 621.08 & 701.4 & 677.79 & $4.24 \%$ & 701.8 & 682.00 & $4.29 \%$ \\
\hline 1500-a-1-d-0_25-e-0_25.stp & 1377.0 & 1376.6 & 1362.93 & $1377.0^{*}$ & 1368.65 & $0.03 \%$ & $1377.0 *$ & 1368.56 & $0.03 \%$ \\
\hline $1000-\mathrm{a}-0 \_6-\mathrm{d}-0.5-\mathrm{e}-0 \_25$.stp & 522.5 & 439.7 & 361.10 & 501.8 & 435.81 & $14.11 \%$ & 514.8 & 449.79 & $17.08 \%$ \\
\hline 1000-a-0_6-d-0_25-e-0_5.stp & 1872.3 & 1866.3 & 1847.82 & $1872.3^{*}$ & 1863.33 & $0.32 \%$ & $1872.3 *$ & 1863.36 & $0.32 \%$ \\
\hline $1000-\mathrm{a}-0-6-\mathrm{d}-0-25-\mathrm{e}-0-25 . \mathrm{stp}$ & 931.5 & $\begin{array}{l}1000.0 \\
875.6\end{array}$ & $\begin{array}{l}1041.02 \\
800.01\end{array}$ & 925.1 & $\begin{array}{l}1000.03 \\
886.88\end{array}$ & $5.65 \%$ & 922.2 & $\begin{array}{l}1003.35 \\
892.35\end{array}$ & $5.33 \%$ \\
\hline 750-a-0_647-d-0_25-e-0_5.stp & 1419.8 & 1419.4 & 1398.21 & $1419.8^{*}$ & 1415.41 & $0.03 \%$ & $1419.8 *$ & 1416.14 & $0.03 \%$ \\
\hline $500-\mathrm{a}-0 \_62-\mathrm{d}-0 \_75-\mathrm{e}-0 \_25 . \mathrm{stp}$ & 171.6 & 170.3 & 147.76 & $171.6^{*}$ & 165.63 & $0.81 \%$ & $171.6^{*}$ & 165.80 & $0.81 \%$ \\
\hline $500-\mathrm{a}-0 \_62-\mathrm{d}-0 \_25-\mathrm{e}-0 \_25 . \mathrm{stp}$ & 460.6 & 432.9 & 387.02 & 449.9 & 430.15 & $3.92 \%$ & 448.7 & 431.71 & $3.64 \%$ \\
\hline 1000-a-0_6-d-0_75-e-0_25.stp & 332.8 & 324.2 & 289.04 & 332.5 & 309.49 & $2.57 \%$ & $332.8^{*}$ & 313.28 & $2.66 \%$ \\
\hline $500-\mathrm{a}-0-62-\mathrm{d}-0-25-\mathrm{e}-0-5 . s t \mathrm{p}$ & 993.0 & 986.4 & 961.43 & $993.0^{*}$ & 982.66 & $0.66 \%$ & $993.0^{*}$ & 983.59 & $0.66 \%$ \\
\hline $1000-\mathrm{a}-1-\mathrm{d}-0 \_25-\mathrm{e}-0 \_75$. stp & 2789.6 & $2789.6^{*}$ & 2789.57 & $2789.6^{*}$ & 2789.58 & $30.00 \%$ & $2789.6^{*}$ & 2789.58 & $0.00 \%$ \\
\hline 1000-a-0_6-d-0_25-e-0_75.stp & 2789.6 & $2789.6^{*}$ & 2787.48 & $2789.6^{*}$ & 2789.58 & $0.00 \%$ & $2789.6^{*}$ & 2789.56 & $0.00 \%$ \\
\hline 1500-a-0_6-d-0_75-e-0_75.stp & 1423.6 & 1417.2 & 1388.62 & $1423.6 *$ & 1411.81 & $0.45 \%$ & $1423.6 *$ & 1419.14 & $0.45 \%$ \\
\hline 1000-a-1-d-0_5-e-0_75.stp & 1770.3 & $1770.3^{*}$ & 1770.28 & $1770.3^{*}$ & 1770.28 & $0.00 \%$ & $1770.3^{*}$ & 1770.28 & $0.00 \%$ \\
\hline 1000-a-0_6-d-0_5-e-0_75.stp & 1762.7 & $1762.7^{*}$ & 1753.70 & $1762.7 *$ & 1757.67 & $0.00 \%$ & $1762.7 *$ & 1757.59 & $0.00 \%$ \\
\hline $1500-\mathrm{a}-1-\mathrm{d}-0-75-\mathrm{e}-0-75 . \mathrm{stp} p$ & 1423.6 & $1423.6^{*}$ & 1423.55 & $1423.6^{*}$ & 1423.61 & $0.00 \%$ & $1423.6^{*}$ & 1423.61 & $0.00 \%$ \\
\hline 1500-a-1-d-0_75-e-0_5.stp & 1089.8 & $1089.8^{*}$ & 1084.94 & $1089.8^{*}$ & 1089.31 & $0.00 \%$ & $1089.8^{*}$ & 1089.50 & $0.00 \%$ \\
\hline 1000-a-1-d-0_25-e-0_5.stp & 1883.2 & $1883.2^{*}$ & 1881.13 & $1883.2 *$ & 1883.21 & $0.00 \%$ & $1883.2^{*}$ & 1883.21 & $0.00 \%$ \\
\hline 1500-a-0_6-d-0_5-e-0_25.stp & 847.5 & 737.0 & 651.80 & $\mathbf{7 8 2 . 4}$ & 728.18 & $6.16 \%$ & 797.2 & 745.30 & $8.17 \%$ \\
\hline 1500-a-0_6-d-0_75-e-0_5.stp & 1089.8 & 1073.5 & 1033.04 & $1089.8^{*}$ & 1074.27 & $1.52 \%$ & $1089.8 *$ & 1081.21 & $1.52 \%$ \\
\hline $750-\mathrm{a}-1-\mathrm{d}-0-25-\mathrm{e}-0-75 . \mathrm{stp}$ & 2116.6 & $2116.6^{*}$ & 2116.58 & $2116.6^{*}$ & 2116.58 & $0.00 \%$ & $2116.6^{*}$ & 2116.58 & $0.00 \%$ \\
\hline 750-a-0_647-d-0_25-e-0_75.stp & 2116.6 & $2116.6^{*}$ & 2114.61 & $2116.6^{*}$ & 2116.58 & $30.00 \%$ & $2116.6^{*}$ & 2116.58 & $0.00 \%$ \\
\hline Average & & & & & & $2.09 \%$ & & & $2.20 \%$ \\
\hline
\end{tabular}

To conclude, on these 32 representative MWCS instances, the swap-vertex move operator alone contributes significantly to the effectiveness of the proposed algorithm, while the auxiliary evaluation function is not so important as for the SPG and the PCSPG instances. One possible reason is that on these MWCS instances, different vertices generally have different weights, thus swapping a pair of vertices rarely leads to a neighboring solution with a $\Delta=0$, making the auxiliary evaluation function irrelevant (actually, this is also the case for the last 8 PCSPG instances of Table 2).

\subsection{Performance on the 11th DIMACS Implementation Challenge}

By combining the techniques described in this work with several other strategies (e.g., tabu search, knowledge learning mechanisms, and adaptive perturbation strategies), we proposed KTS, a knowledge guided tabu search algorithm [28] for the 11th DIMACS implementation challenge dedicated to the broadly defined Steiner tree problems [31]. We took part in the competition on two STP variants, i.e., the RPCST and the PCSPG respectively, which attracted the largest number of participants among the seven competition variants. For each variant, we participated in four main challenge subcategories involving all the competing algorithms, i.e., two Primal Integral subcategories regarding both the solution quality and runtime, and two Primal Bound subcategories regarding only the solution quality. Given that our KTS algorithm is a heuris- 
tic which runs only in sequential mode, we are not concerned by the special subcategories focused on exact algorithms or parallelized algorithms.

Under the competition rules, the KTS algorithm achieved the following performances on the eight subcategories in which it was involved ${ }^{4}$. On the RPCST, it won both the two Primal Integral subcategories, and was tied for the first place on both the two Primal Bound subcategories. On the PCSPG, it was ranked first on one Primal Integral subcategory, and third on the remaining three subcategories. Additionally, although we did not participate in the competition on the SPG (due to time reason), the KTS algorithm yielded remarkable results on several challenging SPG instances, with respect to the current best known results.

Actually, as key elements of the KTS algorithm, the swap-vertex move operator and the auxiliary evaluation function contributed significantly to the achieved performance on the 12 difficult PCSPG instances of groups $\mathrm{H}$, PUCNU and ACTMODPC, including 10 instances with strictly uniform edge costs and 2 instances with nearly uniform edge costs. When these two components are disabled from the KTS algorithm, the performance decreases drastically on these 12 instances. Given the applicability condition of the swap-vertex move operator, it was not applied by KTS to solve the remaining challenge instances with significantly varying edge costs or of too large size (with more than 6000 vertices).

\section{Conclusions}

In this paper, we developed an effective swap-vertex move operator for tackling broadly defined Steiner tree problems (STPs). This operator complements the existing conventional move operators and could be adapted to solve many STP instances (corresponding to various Steiner tree problems), where 1) the optimal solution must be a minimum spanning tree, 2) each edge is associated with a uniform cost (this requirement can be relaxed to include instances with nearly uniform edge costs, using the techniques detailed in [28]), and 3) the input graph is of reasonable size (depending on the available memory of the used computer). We showed that with the aid of dynamic data structures, one can efficiently evaluate the feasibility of every possible swap-vertex move. Additionally, we designed an auxiliary evaluation function, which is able to discriminate different swap moves leading to the same move gain, in order to guide the search towards promising search regions. Our computational study carried out on three representative STP variants (i.e., the SPG, the PCSPG and the MWCS, all being part of the 11th DIMACS implementation challenge) demonstrated the usefulness of the swap-vertex operator and the auxiliary evaluation function for improved search performances. More generally, these techniques could be advantageously combined with other powerful search strategies to create effective algorithms for solving various Steiner tree problems.

${ }^{4}$ Details at: http://dimacs11.zib.de/contest/results/results.html 


\section{Acknowledgments}

We are grateful to the referees and the editors for their insightful comments and suggestions. This work was partially supported by the following projects: LigeRO (2009-2014, Pays de la Loire Region), PGMO (2014-2015, Jacques Hadamard Mathematical Foundation), and a Post-doc fellowship from Angers Loire Metropole. We would like to thank the organizers of the 11th DIMACS Implementation Challenge for organizing the competition and the workshop.

\section{References}

1. Cheng, X., Du, D. Z., Steiner trees in Industry. Springer, 2002.

2. Hakimi, S. L., Steiner's problem in graphs. Networks, 1, 113-133, 1971.

3. Hwang, F. K., On steiner minimal trees with rectilinear distance. SIAM Journal on Applied Mathmatics, 30(1), 104-114, 1976.

4. Smith, W. D., How to find Steiner minimal trees in Euclidean d-space. Algorithmica, $7(2 / 3), 137-177,1992$.

5. Johnson, D., Minkoff, M., Phillips, S., The prize collecting steiner tree problem: theory and practice. Proceedings of the eleventh annual ACM-SIAM symposium on Discrete algorithms, Society for Industrial and Applied Mathematics, Philadelphia, PA, USA, 760769, 2000.

6. Segev, A., The node weighted Steiner tree problem. Networks, 17, 1-17, 1987.

7. Álvarez-Miranda, E., Ljubic, I., Mutzel, P., The maximum weight connected subgraph problem. In: Jünger, M., Reinelt, G. (eds.) Facets of Combinatorial Optimization, Springer, pp. 245-270, 2013.

8. Ferreira, C. E., Filho, F. M. O., Some formulations for the group Steiner tree problem. Electronic Notes in Discrete Mathematics, 18(1), 127-132, 2004.

9. Salazar, J. J., A note on the generalized steiner tree polytope. Discrete Applied Mathematics, 100(1-2), 137-144, 2000.

10. Chen, S. Y., Li, C. F., Chang, Y. W., Yang, C. L., Obstacle-avoiding rectilinear Steiner tree construction based on spanning graphs. IEEE Transactions on Computer-Aided Design of Integrated Circuits and Systems, 27(4), 643-653, 2008.

11. Charikar, M., Chekuri, C., Cheung, T., Dai, Z., Goel, A., Guha, S., Li, M., Approximation algorithms for directed Steiner problems. Journal of Algorithms, 33(1), 73-91, 1999.

12. Imase, M., Waxman, B. M., Dynamic Steiner tree problem. SIAM Journal on Discrete Mathematics, 4(3), 369-384, 1991.

13. Kurz, D., Mutzel, P., Zey, B., Parameterized algorithms for stochastic Steiner tree problems. Mathematical and Engineering Methods in Computer Science, 7721, 143-154, 2013.

14. Vo $\beta$, S., The Steiner tree problem with hop constraints. Annals of Operations Research, 86, 271-294, 1999.

15. Fu, Z. H., Hao, J. K., Breakout local search for the Steiner tree problem with revenue, budget and hop constraints. European Journal of Operational Research, 232(1), 209-220, 2014.

16. Fu, Z. H., Hao, J. K., Dynamic programming driven memetic search for the Steiner tree problem with revenue, budget and hop constraints. INFORMS Journal on Computing, $27(2), 221-237,2015$.

17. Karp, R. M., Reducibility among combinatorial problems. Miller, R. E., Thatcher, J. W., eds. Complexity of Computer Computations, New York, 1972.

18. Garey, M. R., Johnson, D. S., Computers and intractability: a guide to the theory of NP-completeness. Freeman, San Francisco, CA, 1979.

19. Shi, W., Su, C., The rectilinear Steiner arborescence problem is NP-complete. SIAM Journal on Computing, 35(3), 729-740, 2006. 
20. Fischetti, M., Leitner, M., Ljubic, I., Luipersbeck, M., Monaci, M., Resch, M., Salvagnin, D., Sinnl, M., Thinning out Steiner trees: a node-based model for uniform edge costs. Workshop of the 11th Dimacs Implementation Challenge, Providence, Rhode Island, December 4-5, 2014, available online at http://dimacs11.zib.de/workshop.html

21. Gamrath, G., Koch, T., Maher, S. T., Rehfeldt, D., Shinano, Y., SCIP-Jack - A solver for STP and variants with parallelization extensions. Workshop of the 11th Dimacs Implementation Challenge, Providence, Rhode Island, December 4-5, 2014, available online at http://dimacs11.zib.de/workshop.html

22. Fonseca, R., Brazil, M., Winter, P., Zachariasen, M., Faster exact algorithms for computing Steiner trees in higher dimensional Euclidean spaces. Workshop of the 11th Dimacs Implementation Challenge, Providence, Rhode Island, December 4-5, 2014, available online at http://dimacs11.zib.de/workshop.html

23. El-Kebir, M., Klau, G. W., Solving the maximum-weight connected subgraph problem to optimality. Workshop of the 11th Dimacs Implementation Challenge, Providence, Rhode Island, December 4-5, 2014, available online at http://dimacs11.zib.de/workshop.html

24. Minoux, M., Efficient greedy heuristics for Steiner tree problems using reoptimization and supermodularity. INFOR, 28, 221-233, 1990.

25. Uchoa, E., Werneck, R. F., Fast local search for Steiner trees in graphs. ACM Journal of Experimental Algorithms, 17(2), 2.2:1-2.2:22, 2012.

26. Pajor, T., Uchoa, E., Werneck, R., A robust and scalable algorithm for the Steiner problem in graphs. Workshop of the 11th Dimacs Implementation Challenge, Providence, Rhode Island, December 4-5, 2014, available online at http://dimacs11.zib.de/ workshop.html

27. Canuto, S. A., Resende, M. G. C., Ribeiro, C. C., Local search with perturbations for the prize-collecting Steiner tree problem in graphs. Networks, 38, 50-58, 2001.

28. Fu, Z. H., Hao, J. K., Knowledge guided tabu search for the prize-collecting Steiner tree problem in graphs. Workshop of the 11th Dimacs Implementation Challenge, Providence, Rhode Island, December 4-5, 2014, available online at http://dimacs11.zib.de/ workshop.html

29. Burdakov, O., Kvarnstrom, J., Doherty, P., Local search heuristics for hop-constrained directed Steiner tree problem. Workshop of the 11th Dimacs Implementation Challenge, Providence, Rhode Island, December 4-5, 2014, available online at http://dimacs11.zib. de/workshop.html

30. Hoos, H.H., Stützle T., Stochastic Local Search: Foundations and Applications. Morgan Kaufmann, 2005.

31. Johnson, D. S, Koch, T., Werneck, R. F., Zachariasen, M., 11th DIMACS Implementation Challenge in collaboration with ICERM: Steiner tree problems. http: //dimacs11.zib.de, 2013-2014.

32. Dittrich, M. T., Klau, G. W., Rosenwald, A., Dandekar, T., Müller, T., Identifying functional modules in protein-protein interaction networks: an intergrated exact approach. Bioinformatics, 24(13), i223-31, 2008.

33. Rodriguez-Tello, E., Hao, J. K., Torres-Jimenez, J., An improved Simulated Annealing algorithm for bandwidth minimization. European Journal of Operational Research, 185(3): 1319-1335, 2008

34. Rodriguez-Tello, E., Hao, J. K., Torres-Jimenez, J., An effective two-stage simulated annealing algorithm for the minimum linear arrangement problem. Computers \& Operations Research, 35(10), 3331-3346, 2008. 